\title{
La Protesta en Redes Sociales como herramienta para los movimientos sociales ${ }^{1}$
}

\section{The Protest in Social Networks as a tool for social movements}

\author{
Rocío Briceño G. \\ Departamento de Antropología, Universidad de Chile \\ https://orcid.org/0000-0002-4231-6033 \\ rocio.briceno@ug.uchile.cl
}

\begin{abstract}
Resumen
El objetivo de este artículo es conocer las opiniones de personas de diferentes edades y géneros con respecto al rol que juegan las Redes Sociales como facilitadoras, organizadoras y/o difusoras de la protesta social. La metodología utilizada contempló la aplicación de encuestas online a 37 personas, cuyas respuestas fueron analizadas a través del software Atlas.ti. Dentro de los resultados más relevantes se pudo encontrar que las personas tienen una opinión en general positiva de las Redes Sociales, aunque bastante crítica, pues comprenden, al menos superficialmente, los riesgos que contempla su masividad.
\end{abstract}

Palabras clave: redes sociales, movimientos sociales, protesta social, activismo digital

\begin{abstract}
The objective of this article is to know the opinions of people of different ages and genders about the role played by Social Networks as facilitators, organizers and/or disseminators of social protest. The methodology used contemplated the application of online surveys to 37 people, whose answers were analyzed through Atlas.ti software. Among the most relevant results it was found that people have, in general, a positive opinion of Social Networks, although quite critical, because they understand, at least superficially, the risks that their massiveness contemplates.
\end{abstract}

Keywords: social networks, social movements, digital protest, digital activism

\footnotetext{
${ }^{1}$ Nota: Este trabajo de investigación se realizó dentro de la asignatura "Software para análisis de datos cualitativos" en el primer semestre de 2019.
} 


\section{Introducción}

El uso de Redes Sociales por parte de la sociedad se ha masificado. En la actualidad, la gran mayoría de las personas utiliza al menos una red social y su uso es tan diverso como las personas que la utilizan. Por lo mismo, el uso de Redes Sociales ha sido tanto valorado como cuestionado con el tiempo. Entre sus aspectos positivos podemos destacar su utilidad para difundir información urgente, contactarse con personas de otras latitudes y, principalmente, democratizar el acceso a la información y la posibilidad de expresar la opinión, en otras palabras,

"las tecnologías digitales han facilitado que usuarios de todo el mundo puedan relacionarse y compartir opiniones y experiencias; los internautas tienen identidad virtual, que desarrollan a través del conjunto de plataformas que suponen los «social media». Estos nuevos canales han cambiado los parámetros de la comunicación entre individuos y colectivos, permitiendo que el diálogo se democratice y multiplique exponencialmente" (García, del Hoyo, y Fernández, 2014: 36)

Sin embargo, esos mismos aspectos positivos pueden convertirse en un riesgo, pues fácilmente se puede difundir información falsa. Un ejemplo de ello son las conocidas fake news, las mismas que tanta polémica causaron en las últimas elecciones presidenciales de Estados Unidos. Por lo anterior, es que comprender los efectos que estas plataformas tienen sobre la protesta y movilizaciones sociales se hace tan relevante.

Son varios los estudios que han tratado esta temática, sin embargo, siempre se le ha puesto atención al uso que le da la población más joven, en detrimento de otras edades como, por ejemplo, las personas mayores, aun cuando el contexto global señala que este segmento de la población crece aceleradamente:

"De manera habitual, se señala que las redes sociales son un fenómeno juvenil, y los estudios dan buena cuenta de la importancia que ha alcanzado el fenómeno de las redes sociales de Internet, redes cuya primera función, como advierte su propio nombre, es la de facilitar la interrelación" (García y del Hoyo, 2013: 113)

Por ello, si bien las Redes Sociales y el uso de Internet sigue siendo un fenómeno principalmente juvenil, comprender el impacto en las formas de relacionarse e informarse entre personas de todas las edades, se vuelve relevante. En ese sentido, considero que las Redes Sociales no sólo son una expresión de la forma de relacionarse, sino que son capaces de incidir en ésta, formando movimientos de masas con objetivos comunes, permitiendo su organización y, en consecuencia, la proliferación de protestas sociales dentro del espacio digital, fenómeno conocido como activismo digital, el cual, en ocasiones, puede salir de ese espacio.

Por lo anterior, en la presente investigación interesa comprender cuál es el papel que otorgan personas de diferentes edades a las Redes Sociales en tanto facilitadoras, organizadoras y/o 
difusoras de la protesta social, comprendiendo la necesidad de reconocer las consecuencias y el alcances que tienen dichas Redes. Por lo mismo, diferentes autores abogan por una alfabetización digital (Rovira, 2013; García y del Hoyo, 2013; García, del Hoyo, y Fernández, 2014), en el sentido de que se debe educar sobre el alcance y los riesgos de los medios de comunicación digitales a todos los usuarios y usuarias de éstos, sin importar si se trata de "nativos digitales" o de otras generaciones.

\section{Metodología}

La presente investigación corresponde a un estudio de carácter exploratorio (Sampieri, Fernández, \& Baptista, 2010), puesto que busca dar cuenta de las opiniones sobre el uso de Redes Sociales en relación con la protesta, de diferentes actores de la sociedad. Como se expresó anteriormente, la mayor parte de los estudios en esta línea se enfocan en el papel de los jóvenes. Por lo mismo, en esta investigación se quiso dar cuenta de la opinión de personas de diferentes edades para comprender su posición.

El instrumento de levantamiento de datos utilizado fue el cuestionario (Asún, 2006), se diseñó una única forma, la cual sería aplicada indistintamente a toda persona que quisiera contestar la encuesta. Las preguntas (ver Tabla 1) se dividieron en dos secciones: la primera de caracterización, la cual contó con 3 preguntas cerradas, 1 semiabierta (Género) y 1 abierta (¿Qué Redes Sociales utiliza?). La segunda sección buscaba conocer la opinión de los y las encuestadas, por lo que contó exclusivamente con preguntas abiertas.

Tabla 1: Cuestionario

\begin{tabular}{|l|}
\hline A. Preguntas de caracterización: \\
\hline 1. Género \\
\hline 2. Edad \\
\hline 3. Tipo de colegio en que estudió \\
\hline 4. ¿Ha participado de algún movimiento y/o protesta social? \\
\hline 5. ¿Qué Redes Sociales utiliza? \\
\hline B. Preguntas de opinión: \\
\hline $\begin{array}{l}\text { 1. ¿Crees que las Redes Sociales son una herramienta útil para } \\
\text { 1a protesta social? }\end{array}$ \\
\hline 2. ¿Por qué crees eso? \\
\hline
\end{tabular}




\begin{tabular}{|l|}
\hline $\begin{array}{l}\text { 3. ¿Cómo pueden las Redes Sociales ayudar a } \\
\text { levantar/organizar/difundir una protesta o movimiento social? }\end{array}$ \\
\hline $\begin{array}{l}\text { 4. ¿Has sentido ganas de participar en una protesta o } \\
\text { movimiento por haberlo visto a través de tus Redes Sociales? } \\
\text { Cuenta tu experiencia }\end{array}$ \\
$\begin{array}{l}\text { 5. ¿Qué efectos podrían tener las Redes Sociales sobre las } \\
\text { protestas o movimiento sociales? Cuenta aspectos negativos y } \\
\text { positivos }\end{array}$ \\
\hline
\end{tabular}

La herramienta para aplicar dicho cuestionario fue una encuesta on-line, a través del servicio ofrecido por Google llamado Formularios de Google. De esta manera, a través de un enlace, toda persona que quisiera responder podría hacerlo. El enlace fue difundido a través de diferentes grupos de Facebook y WhatsApp, así como también a través de correo electrónico y estuvo disponible entre los meses de Abril y Mayo de 2019. Como la investigación perseguía conocer la opinión de personas de diferentes edades, el enlace fue difundido con un mensaje que invitaba a aplicar la encuesta a otras personas que pudieran no tener acceso al enlace, por ejemplo, las personas mayores pertenecientes a la familia.

\section{Muestra}

Se recibieron un total de 37 respuestas. Originalmente no se establecieron cuotas ni características específicas para la muestra, pues debía ser lo más heterogénea posible, considerando el alcance que puede tener un enlace web y el período de tiempo para su aplicación.
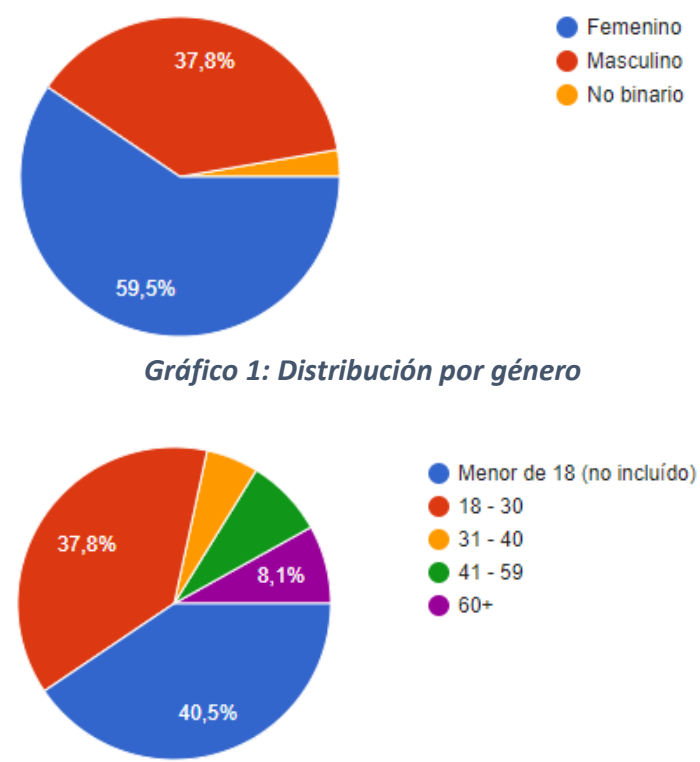

Gráfico 2: Distribución por edad
Finalmente, la muestra quedó definida mayoritariamente por personas de género femenino (59,5\%), menores de 18 años (40,5\%), provenientes de establecimientos particulares subvencionados $(48,6 \%)$ y que han participado, al menos una vez, en movimientos y/o protestas sociales $(62,2 \%)$. Además, sobre el uso de Redes Sociales, las más utilizadas fueron: WhatsApp $(97,3 \%)$, Instagram $(75,7 \%)$ y Facebook $(62,2 \%)$.

Por último, de las 37 encuestas respondidas, 36 fueron procesadas, eliminando una de ellas en que no se respondía a las preguntas de opinión. Para el análisis del material recolectado se utilizó el software Atlas.ti, poniendo énfasis en 
las preguntas de la sección “opinión” y relacionándolas con las de caracterización. Por ello, el análisis se dividirá en 4 secciones para poder describir el material obtenido para cada pregunta de opinión.

\section{Análisis}

\section{Utilidad de las Redes Sociales}

Esta sección corresponde a la justificación de la pregunta cerrada " Crees que la protesta social por RRSS es útil?", cuyas posibles respuestas eran: Sí (62,2\%), No (2,7\%), A veces $(35,1 \%)$.

Existe clara tendencia, sin diferencias entre edades, géneros y establecimientos educacionales, a considerar que la protesta es útil, al menos en ocasiones, porque permite difundir información a un gran número de personas. Esto apunta, generalmente, a la organización de marchas y protestas basadas en la colectivización de demandas y/o petitorios.

Junto con la anterior, aunque en menor medida, hay personas que piensan que la utilidad de la protesta social en RRSS radica en la rapidez de la difusión y la posibilidad de organizar fácilmente dichas protestas, así como también en la posibilidad de expresar libremente nuestras ideas y alcanzar a un gran número de personas con diversas cualidades (público heterogéneo), a quienes muchas veces se les hace más fácil acceder a la información a través de RRSS.

Por último, una menor cantidad de personas hizo alusión a la inutilidad de la protesta en RRSS por quedarse encasillada en lo virtual y no realizar cambios concretos en la sociedad. En contraposición a esto, hay personas que creen que las RRSS pueden crear vínculos entre quienes tienen intereses afines y, por lo tanto, fomentar la comunicación. Así también, algunas personas manifestaron sentirse apoyadas por otras gracias a la difusión de demandas en RRSS.

Por último, algunos aspectos negativos fueron destacados, por ejemplo, que se puede tergiversar muy fácilmente la información encontrada; que no es una protesta sustentada en un deseo real de cambiar algo, sino en "protestar por protestar" o que muchas veces se sienten presionados por la multitud y la protesta se transforma en un "compromiso social", es decir, no en un interés generado a partir de una reflexión individual, sino más bien en un movimiento de masas.

\section{Las Redes Sociales como herramienta para organizar y difundir la protesta social}

Ante la pregunta "¿Cómo pueden las Redes Sociales ayudar a levantar/organizar/difundir una protesta o movimiento social?" la mayoría de las personas se refirió a la gran cantidad de personas que pueden acceder a la información sobre protestas sociales a través de las RRSS, gracias a ello se logra efectivamente convocar a un gran número para realizar 
protestas, es decir, aparentemente se logra sacar el activismo digital a la calle. No obstante, si bien las y los encuestados expresaron que, a través de las RRSS, se pueden organizar fácilmente manifestaciones, algunos también explicaron que esta organización sólo se puede realizar "cara a cara", por lo que la discusión a través de medios digitales se vuelve inútil. Esto tiene relación con un factor que fue expresado tanto positiva como negativamente, y es que las RRSS generan una comunidad virtual que muchas veces ayuda a convocar

Ilustración 1: Aspectos más mencionados por personas provenientes de establecimientos municipales

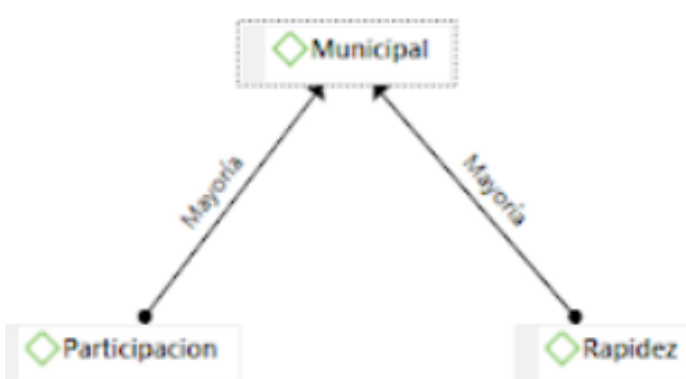

gente diversa, pero otras veces puede generar el estancamiento de la protesta al no poder traspasar la pantalla.

Otro aspecto relevante se refiere a la rapidez (nombrado principalmente por las personas provenientes de colegios Municipales) con que circula la información en RRSS, destacándolo como un factor positivo.

Por último, un menor número de personas hicieron referencia a que las RRSS ayudan a la organización y difusión de la protestas social en tanto brindan accesibilidad y la libertad de opinar a personas que pueden no tener esa facilidad en el mundo no-virtual. En este punto, se hace especial énfasis en la creación de redes con intereses afines que fomentan la comunicación y que pueden utilizar diversos medios para visibilizar su malestar u opinión.

\section{Las Redes Sociales como incentivo a la participación ciudadana}

Ante la pregunta "¿Has sentido ganas de participar en una protesta o movimiento por haberlo visto a través de tus Redes Sociales?" las respuestas brindadas por los y las encuestadas fueron muy diversas, por lo que no se puede hablar de una tendencia clara. No obstante, un gran número manifestó no haber sentido ganas de participar en una protesta por haberla visto en RRSS.

Dentro de las personas que sí manifestaron ganas -aunque no hubieran participado efectivamente-, los motivos se construían principalmente en contraposición a la (des)información proveniente de los medios de comunicación tradicionales (principalmente noticiarios de televisión), pero también consideraban un incentivo la información e imágenes que se suben a las RRSS después de las protestas y, por lo tanto, la publicidad que se hace de éstas, lo cual va generando una especie de "presión social" (ya mencionada anteriormente no sólo como un aspecto positivo) y ganas colectivas de "ser parte" de un cambio social.

Sin embargo, algunas personas también hicieron alusión al peligro que estas protestas pueden representar, por ejemplo, la concurrencia a la protesta de grupos que no responden al objetivo principal y acuden sólo para realizar desmanes, poniendo -aparentemente- en peligro a los y 
las asistentes. Este aspecto fue mayormente comentado por personas menores de edad y provenientes de colegios particulares subvencionados.

Ilustración 2: Aspectos más mencionados por personas provenientes de establecimientos particulares subvencionados

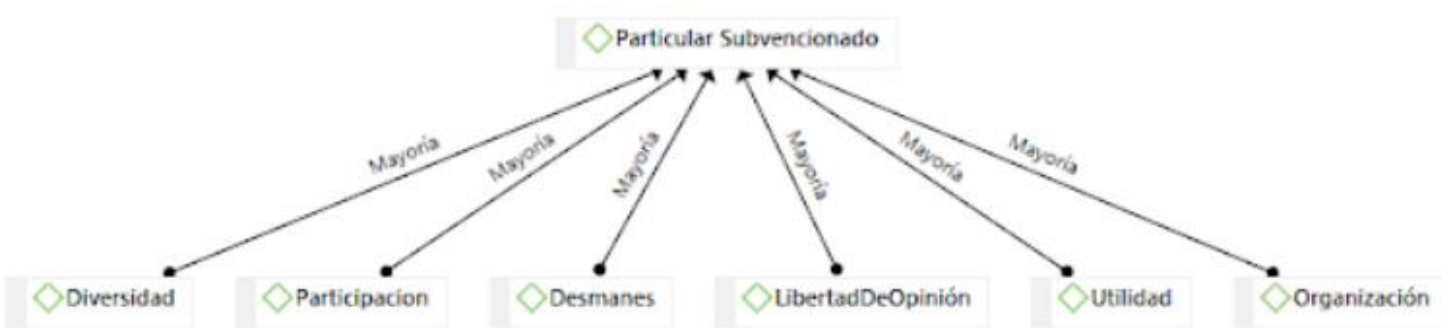

4. Efectos positivos y negativos de las Redes Sociales sobre las protestas y movimientos sociales

La pregunta "¿Qué efectos podrían tener las RRSS sobre las protestas o movimientos sociales?" sirve como un resumen y confirmación de las reflexiones realizadas con anterioridad. De esa manera, los aspectos positivos que más fueron mencionados son: la masividad y gran capacidad de difusión de la protesta a través de las RRSS.

En menor cantidad, las personas creen que un aspecto positivo es la inmediatez con que podemos acceder a la información, así como también la posibilidad de dar opiniones libremente y que éstas tengan cabida en personas muy diversas.

Muy pocas personas creen que la protesta en RRSS puede generar cambios sociales, la posibilidad de comunicarnos y/o de sentir que somos parte de un movimiento mayor (pertenencia).

Por otro lado, los principales aspectos negativos destacados fueron la posibilidad de desinformar más que informar gracias a las RRSS, es decir, que muchas veces la información se puede tergiversar y crear opiniones falsas, estos aspectos fueron mencionados principalmente por personas entre 31 y 59 años. Por lo mismo, también un gran número de personas, explicaron que, en tanto la información en RRSS puede ser falsa, resulta poco creíble.

Ilustración 3: Aspectos más mencionados por personas de entre 31 y 59 años

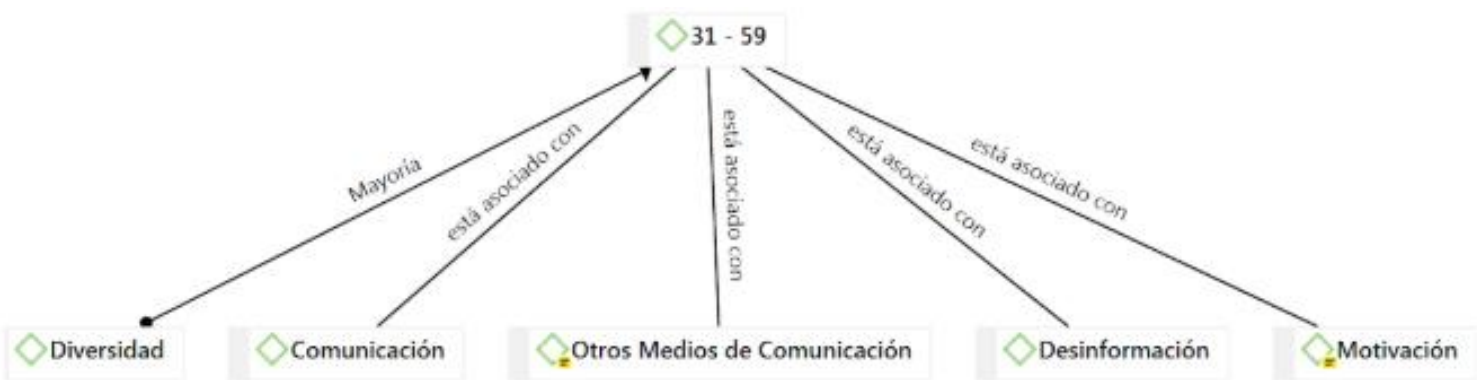


Una menor cantidad de personas piensa que aspectos negativos pueden ser el peligro al que nos encontramos expuestos en y por las RRSS, por ejemplo, la convocatoria de personas con otros fines en las manifestaciones, lo que puede provocar muchos desmanes y, por lo tanto, una exposición insegura (mencionado principalmente por personas menores de edad). Lo mismo corre a nivel virtual, puesto que nuestras opiniones y datos son compartidos con una gran cantidad de personas y no podemos filtrar a quienes llegan, esto también fue dicho por personas para referirse a la infiltración de fuerzas policiales en grupos virtuales que buscan organizarse para discutir sobre manifestaciones sociales.

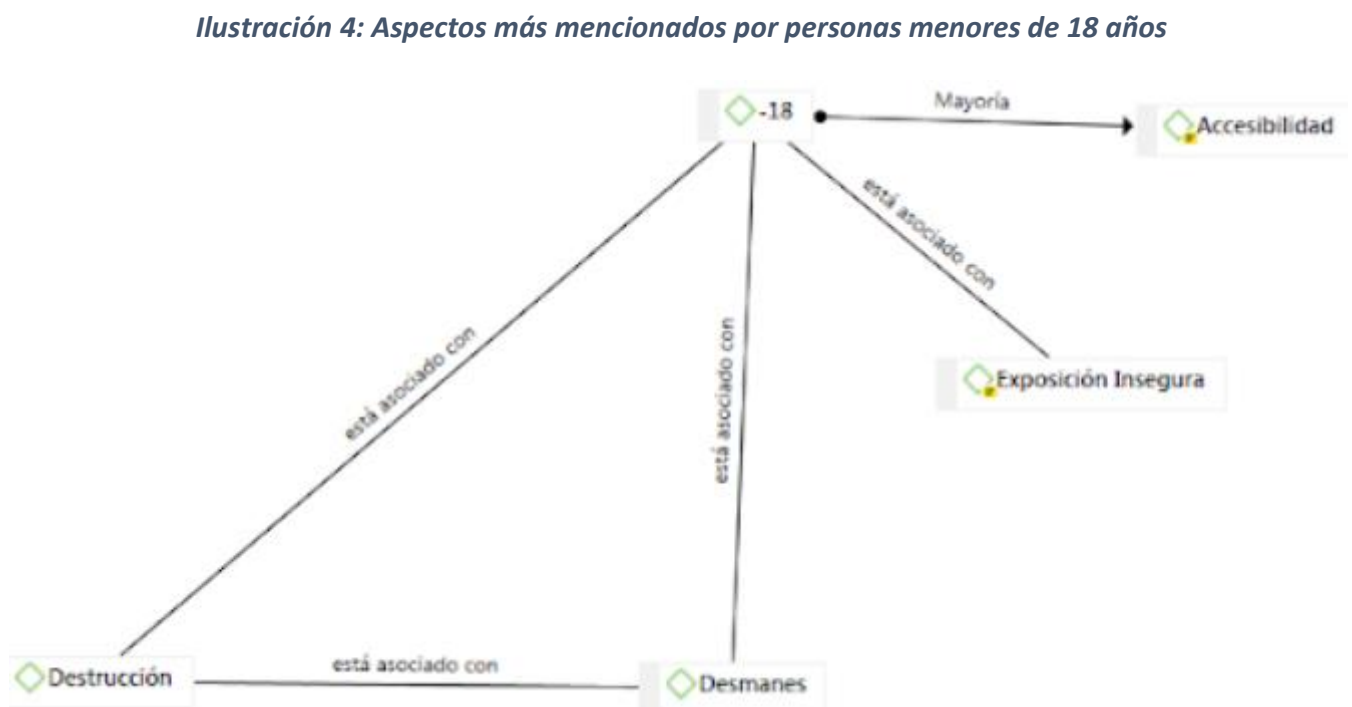

\section{Conclusión}

A partir de la información obtenida podemos notar que, en general, existe una valoración positiva del uso de Redes Sociales, sobre todo porque las personas reconocen su utilidad para organizar y difundir protestas sociales incluso fuera del espacio digital. No obstante, a pesar de lo que se podría creer, quienes respondieron la encuesta demostraron una crítica que no se esperaba al comienzo de la investigación, en tanto se muestran conscientes de que existen riesgos producto de la misma masividad de las Redes Sociales. Además, mencionaron también la proliferación de información falsa y, por lo tanto, rescataron que muchas veces la información disponible resulta poco creíble.

Como vimos, la variable "colegio de procedencia" no pareció afectar en el tipo de opiniones realizadas (en cuanto a cualidades positivas o negativas), aunque sí en su heterogeneidad, pues en general, las personas de colegios municipales tenían opiniones parecidas y expresadas con conceptos similares, mientras que las personas de colegios particulares y particulares subvencionados expresaron opiniones disímiles.

No se encontraron grandes diferencias entre personas de distintas edades, pero hay que reconocer que el número de encuestados no fue equitativo, por lo que esto pudo resultar en un sesgo que afectó los resultados analizados. De todos modos, resulta interesante que 
prácticamente sólo la sección menor de 18 años comentara sobre los desmanes relacionados con las protestas sociales, mientras que este no fue un aspecto mencionado por la población mayor de 60 años, siendo que lo esperado era justamente lo opuesto. Esto demuestra que aún existen muchos sesgos y prejuicios a la hora de realizar estudios con personas mayores, razón por la cual se vuelve incluso más necesario continuar en esta línea investigativa, sobre todo teniendo en cuenta que hoy existen muchos programas de alfabetización digital dirigidos a esta parte de la población.

\section{Bibliografía}

Asún, R. (2006). Construcción de Cuestionarios y Escalas: El proceso de la producción de información cuantitativa. En Canales, M. (Ed. y Coord.), Metodologías de investigación social. Introducción a los oficios. Santiago: Lom

García, M., y del Hoyo, M. (2013). Redes sociales, un medio para la movilización juvenil. Zer, 18(34): 111-125. Disponible en https://www.ehu.eus/ojs/index.php/Zer/article/view/10649/9887

García, M., del Hoyo, M., y Fernández, C. (2014). Jóvenes comprometidos en la Red: El papel de las redes sociales en la participación social activa. Comunicar, 22(43): $35-43$

Rovira, G. (2013). Activismo mediático y criminalización de la protesta: medios y movimientos sociales en México. Convergencia(61): 35-60. Disponible en http://www.scielo.org.mx/pdf/conver/v20n61/v20n61a2.pdf

Sampieri, R., Fernández, C., y Baptista, M. (2010). Metodología de la investigación (5 ${ }^{\mathrm{a}}$ Edición). México D.F.: Mc Graw Hill 